\title{
Arbor
}

\section{Ciencia y Tecnología de alimentos}

\section{Daniel Ramón Vidal}

Arbor CLXVI, 653 (Mayo 2000), 127-139 pp.

En este artículo se presenta la composición y actividades del área de Ciencia y Tecnología de alimentos del Consejo Superior de Investigaciones Científicas. Asimismo, se comentan las distintas líneas de investigación y se discuten posibles estrategias científicas de futuro.

Una constante fisiológica de cualquier ser vivo es su necesidad de alimentarse. A este respecto, la diferencia entre el hombre y las demás especies que puebla nuestro planeta es doble: por un lado nos hemos convertido en productores de alimentos y por otro hemos hecho de la alimentación algo más que un simple ejercicio de mantenimiento de los niveles energéticos, llegando a convertirla en un fenómeno cultural. Desde que el hombre se hizo agricultor hace más de catorce mil años investigamos la producción, transformación y elaboración de alimentos. Durante una gran parte de este tiempo la investigación fue totalmente empírica, se basaba en pruebas de acierto o error y carecía de base científica. Tras esta larga época, a comienzos de este siglo se aplicó el método científico al estudio de los alimentos, dando lugar al nacimiento de la disciplina científica denominada Ciencia y Tecnología de alimentos. Aunque el objeto de este artículo no es su estudio, conviene recordar que durante todos estos años apenas ha habido desarrollos científicos propios de la misma. Por el contrario, esta disciplina científica se ha nutrido de desarrollos producidos en otros campos del conocimiento como la química analítica u orgánica, la bioquímica, la microbiología, la genética, la físiología humana o la robótica. Como posteriormente comprobaremos, la consecuencia de este hecho es la necesidad de contar 
con equipos pluridisciplinares de investigación abiertos a cualquier nueva tecnología, por muy distante que la misma parezca estar de la alimentación.

En nuestro país existe un colectivo importante de científicos trabajando en Ciencia y Tecnología de alimentos. Su productividad científica es adecuada y no presenta grandes diferencias de calidad con la de otros grupos europeos. Sin duda, a ello ha contribuido la existencia de programas específicos de Ciencia y Tecnología de alimentos dentro del pasado Plan Nacional de Ciencia y Tecnología y del III y IV Programa Marco de la Unión Europea. Hace más de cincuenta años, un grupo de investigadores del Consejo Superior de Investigaciones Científicas (CSIC) fueron pioneros en España en la investigación en Ciencia y Tecnología de alimentos. Desde entonces el CSIC viene potenciando esta disciplina científica que constituye una de las ocho áreas de investigación del organismo. A lo largo de este artículo comentaré mi opinión personal sobre el presente y el futuro de la misma ${ }^{1}$.

\section{La industria agroalimentaria española}

Antes de hablar de investigación en Ciencia y Tecnología de alimentos es importante conocer la realidad del sector industrial español que, al menos en teoría, debería ser el principal receptor de los desarrollos obtenidos. La actividad agroalimentaria española tiene un peso importante dentro de la economía de nuestro país. Según datos oficiales, en el año 1997 nuestra producción agraria se cifró en 4,6 billones de pesetas con un valor añadido bruto de 2,7 billones de pesetas. Si centramos nuestro análisis en el sector que se ocupa de la transformación en alimentos de estos productos, las cifras aun son más importantes, ya que este representa el $20 \%$ de la industria española y es nuestro primer sector industrial con una producción superior a los 8,6 billones de pesetas. En su conjunto el sector agroalimentario español supone el 7,6 del PIB nacional, da empleo al 11,3\% de la población activa y representa el $15 \%$ de las transacciones de comercio exterior de nuestro país. Sin duda, la importancia de estas cifras demanda la existencia de grupos de investigación de calidad en Ciencia y Tecnología de alimentos.

Como en otros países de la UE, la industria agroalimentaria española se enfrenta a una competencia creciente. Como indica el nuevo Plan Nacional de Investigación ${ }^{2}$, es necesario introducir cambios radicales en la orientación de las producciones primarias y sus transformaciones. 


\section{Ciencia y Tecnología de alimentos}

El objetivo será dar productos que satisfagan a un consumidor con un poder adquisitivo creciente y que se muestra sensible al consumo de productos de excelente calidad. Por desgracia, la mayor parte del sector primario y la industria productiva española tiene un grave problema de atomización de empresas que, dada la falta de capital de las pequeñas y medianas empresas, dificulta la inversión en investigación y desarrollo. Un buen ejemplo de ello lo constituye la dependencia exterior que presenta el sector, sobretodo en tecnologías de producción primaria, de producto y de proceso. Un estudio reciente de la Federación de Alimentos y Bebidas estimaba que la autonomía de renovación de tecnologías de producto en el sector agroalimentario español era del $51,5 \%$ y la de tecnologías de proceso de un $41 \%$. Todo ello da idea de la importancia de una inversión en investigación y desarrollo que, dada la idiosincrasia de la industria agroalimentaria española, deberá hacerse mayoritariamente en centros públicos de investigación. El CSIC aspira a satisfacer sus necesidades y ser un punto de referencia de la industria agroalimentaria española en este objetivo.

\section{Descripción del área de Ciencia y Tecnología de alimentos del CSIC}

El área de Ciencia y Tecnología de alimentos del CSIC tiene como objetivo la investigación de la producción de alimentos. Los distintos grupos del área analizan todos los eslabones de la cadena de producción de un alimento. Para ello se lleva a cabo una investigación básica orientada y pluridisciplinar, con un fuerte contenido en bioquímica biotecnología, microbiología y química y un desarrollo elevado de nuevas técnicas analíticas. Se investiga en una gama variada de productos que incluyen alimentos vegetales (frutas, hortalizas, aceites), animales (leche, productos cárnicos curados, pescado y sus derivados) o fermentados (pan, aceitunas, vino, derivados lácteos).

Como se indicó anteriormente, esta área del conocimiento se inició hace más de cincuenta años, cuando el CSIC creó varios núcleos de investigación en Madrid, Sevilla y Valencia que dieron lugar posteriormente a una red de institutos distribuidos por toda la geografía española. Las investigaciones de estos grupos fueron pioneras en nuestro país, pudiéndose afirmar que la investigación en Ciencia y Tecnología de alimentos en España nació, en gran medida, en el seno del CSIC. Con el paso de los años estos grupos fueron creciendo hasta llegar a la situación actual en la que el área está constituida por ocho institutos, 
seis propios del área y dos compartidos con otras áreas de investigación del CSIC, que se detallan a continuación por orden alfabético de sus siglas:

Centro de Edafología y Biología Aplicada del Segura (CEBAS). Sito en la ciudad de Murcia, es uno de los dos institutos compartidos con otras áreas del conocimiento, en este caso con la de Ciencias Agrarias y la de Recursos Naturales. Dentro del organigrama administrativo del centro existe un Departamento de Tecnología de alimentos donde se adscriben los investigadores del área de Ciencia y Tecnología de alimentos. Las líneas de investigación se centran en alimentos vegetales, y hacen referencia a la conservación y calidad microbiológica de alimentos, la tecnología postcosecha de diversos productos vegetales y la existencia de compuestos de interés nutricional en varios alimentos.

Instituto de Agroquímica y Tecnología de Alimentos (IATA). Está ubicado en el Campus Científico de la Universitat de València. Siendo un instituto propio del área tiene una ampliación de claustro por la que siete profesores de la Universitat de València trabajan desde hace tres años en sus instalaciones. Es un Instituto pluridisciplinar con una fuerte componente de investigación en biotecnología de alimentos, sobre todo en el estudio de distintos microorganismos responsables de la producción de alimentos fermentados (pan, vino, productos cárnicos curados) y aditivos alimentarios, así como en el desarrollo de nuevas técnicas moleculares para el control de procesos y la detección de patógenos. Hay grupos de trabajo en cereales y sus derivados (pan, pastelería), producción de productos cárnicos curados, y tecnología postcosecha de vegetales (fundamentalmente cítricos). También se investiga en el análisis organoléptico y las propiedades físico-químicas de alimentos, la detección de tóxicos, la producción de envases plásticos y el desarrollo de procesos térmicos para la conservación de alimentos. Cuenta con plantas piloto de biotecnología, cereales y derivados, postcosecha, procesos de termoresistencia y productos cárnicos.

Instituto de Fermentaciones Industriales (IFI). Localizado en Madrid, es un instituto propio del área cuyos investigadores llevan a cabo investigaciones de vanguardia en la aplicación de técnicas analíticas en la tecnología de alimentos, la detección de compuestos de interés nutricional en alimentos vegetales, la producción de zumos y la microbiología de diversos alimentos fermentados (productos cárnicos curados, vino).

Instituto del Frío (IFR). Sito en el Campus de la Universidad Complutense de Madrid, es un instituto propio del área cuyas líneas principales de investigación son la producción de derivados lácteos (quesoś) y cárnicos, la obtención de surimi, la tecnología postcosecha de diversas 
frutas y hortalizas, la evaluación nutricional de distintos aditivos alimentarios, y diversas facetas de la ingeniería de procesos alimentarios. Cuenta con una planta piloto de aplicación industrial del frío en procesos agroalimentarios.

Instituto de la Grasa (IGR). Localizado en Sevilla, es un instituto propio del área perfectamente integrado en su entorno socioeconómico al llevar a cabo investigaciones en productos típicos de Andalucía: aceite, aceitunas y fresas. Mantiene líneas de investigación en química y bioquímica de lípidos y pigmentos, tecnología de la producción del aceite y la aceituna de mesa, tecnología postcosecha de la fresa, microbiología de alimentos fermentados y aplicación de técnicas informáticas de vanguardia en el análisis organoléptico de aceites. El instituto cuenta con una almazara experimental y una planta piloto de producción de aceitunas, así como con una instalación semiindustrial para la producción de hidrolizados de proteína a partir de desechos industriales.

Instituto de Investigaciones Marinas (IMI). Ubicado en Vigo, es un instituto compartido con el área de Recursos Naturales. En el organigrama administrativo del instituto existe un Departamento de Tecnología de alimentos donde se integran los científicos del área de Ciencia y Tecnología de alimentos. Las líneas de investigación tratan fundamentalmente sobre los productos de la pesca, si bien, durante los últimos dos años se han abierto líneas de investigación en otros alimentos típicos gallegos. Se investiga en la química y bioquímica de los productos de la pesca, el desarrollo de métodos moleculares para la detección de fraudes en la producción de conservas de pescado, la eliminación de residuos de la industria pesquera, la conservación de pescado y la ingeniería de procesos.

Instituto de Nutrición y Bromatología (INB). Es un centro mixto con el Departamento de nutrición y bromatología de la Universidad Complutense de Madrid, donde tiene localizadas sus instalaciones. Se investiga en inmunonutrición, biodisponibilidad de nutrientes y componentes nutricionales de los aceites.

Instituto de Productos Lácteos de Asturias (IPLA). Sito en la ciudad ovetense de Villaviciosa, es un instituto propio del área con un Patronato formado por el Principado de Asturias, la Universidad de Oviedo, la empresa Central Lechera Asturiana y el CSIC. Se investiga en microbiología y bioquímica de la leche y sus derivados. Tiene un laboratorio de análisis para el exterior que ha establecido excelentes contactos con el sector industrial.

Aparte de los institutos reseñados, el área cuenta con tres investigadores científicos adscritos al área trabajando en institutos de otras 
áreas del conocimiento (Centro de Investigación y Desarrollo, Estación Experimental del Zaidín e Instituto Mediterráneo de Estudios Avanzados). El organigrama del área se completa con la existencia de dos Unidades Asociadas que se describen a continuación:

Colección Española de Cultivos Tipo (CECT). Es un servicio de la Universitat de València que abastece y deposita cultivos microbianos. Es autoridad de depósito internacional a efectos de patente. En abril de 1996 se aprobó la creación de una Unidad Asociada entre la CECT y el IATA. Desde entonces viene funcionando manteniendo proyectos compartidos cuyos resultados han dado lugar a un buen número de publicaciones conjuntas en revistas internacionales de reconocido prestigio.

Departamento de Microbiología de la Universidad de Oviedo. El grupo de Microbiología de bacterias lácticas de la Universidad de Oviedo es Unidad Asociada al IPLA desde junio de 1996. Mantiene proyectos de investigación compartidos con investigadores del IPLA, fruto de los cuales se han publicado varios artículos de investigación en revistas internacionales de reconocido prestigio.

En total, el área está formada por un colectivo de 183 científicos, 244 componentes de la escala de personal de apoyo, más de 50 contratados postdoctorales y un número difícilmente delimitable de doctorandos. Estas cifras no suponen más del 8,5 \% del total de personal científico del CSIC, siendo el área de Ciencia y Tecnología de alimentos la más pequeña de todas las del organismo. Desde el año 1987 en que el área suponía el 9\% del total de personal investigador del CSIC se venía produciendo una lenta reducción de su tamaño. Durante los últimos tres años se ha producido una apuesta decidida del actual equipo directivo del CSIC por esta área del conocimiento que ha contado con el apoyo del Comité Científico Asesor y la Junta de Gobierno. Como más tarde comentaremos, esta apuesta ha pasado por la interacción con otras áreas del conocimiento y la atracción de científicos jóvenes de excelencia. Un análisis de las jubilaciones previstas en los próximos cinco años revela una situación preocupante en el IATA y el INB donde se producirán bajas que afectarán al $25 \%$ y el $40 \%$, respectivamente, del colectivo científico actual.

Durante los últimos años se ha generado una cantera importante de contratados postdoctorales. Como se indicó anteriormente, en la actualidad hay más de 50 contratados financiados por el Ministerio de Educación y Cultura (MEC), el CSIC o fondos de proyectos. Muchos de ellos tienen curricula excelentes y cumplen los requisitos para poder acceder a una plaza de científico titular del CSIC o Profesor Titular 


\section{Ciencia y Tecnología de alimentos}

de Universidad. En el intervalo 1995-98, tan sólo dos de los contratados postdoctorales del área obtuvieron plazas de Profesor Asociado en universidades españolas (Universidad de Murcia y Universitat de València). $\mathrm{Al}$ margen de estos casos, los que han intentado competir por plazas universitarias han sido eliminados, en casi todos los casos por su falta de docencia. Una forma de paliar este problema es intentar implicar a los investigadores del área en la docencia. En la actualidad, muchos de nuestros investigadores participan en docencia reglada de tercer ciclo con diferentes universidades. Merece la pena destacar el caso del IATA cuya participación en la licenciatura de Ciencia y Tecnología de alimentos de la Universitat de València y la Universidad Politécnica de Valencia es intensa, al extremo que 12 de sus investigadores en plantilla son Profesores Asociados de estas dos universidades. También es destacable el papel del IFI en la puesta en marcha de la licenciatura en Ciencia y Tecnología de alimentos de la Universidad Autónoma de Madrid. Lejos de lo que muchos piensan, este tipo de actuaciones no hace sino beneficiar el papel del CSIC en la investigación al permitir incrementar nuestras colaboraciones científicas a la vez que aproximar a nuestras instalaciones jóvenes estudiantes que pueden convertirse en excelentes doctorandos.

En cuanto a las oposiciones del CSIC, de las cuarenta y una plazas de científico titular ofertadas durante el período 1995-99, treinta y seis fueron obtenidas por contratados del área, sin duda un número demasiado elevado que podría indicar una cierta endogamia. El perfil medio de los científicos titulares que han ingresado en el área durante los últimos tres años se corresponde con una edad próxima a los 35 años, una media de dos años de estancia en un laboratorio extranjero, disfrute de contratos postdoctorales por más de dos años, más de veinte publicaciones en revistas internacionales de reconocido prestigio y una patente. Los valores de producción científico-tecnológica de estos jóvenes científicos, hechas las correcciones de tiempo precisas, están por arriba de la media del área, indicando que se está dando entrada a profesionales extraordinariamente formados que, sin duda en tiempo breve, revitalizarán el área.

Si bien el colectivo de investigadores en Ciencia y Tecnología de alimentos del CSIC es el mayor núcleo de investigación en esta disciplina científica en España, y de los mayores en Europa, el bajo número de interacciones científicas entre sus distintos componentes reduce su fortaleza. Si consideramos el global del CSIC, como comentaremos posteriormente, las interacciones con otras áreas del conocimiento del organismo serían un factor estratégico de incremento de calidad y competitividad. 


\section{Las líneas de investigación del área de Ciencia y Tecnología de alimentos del CSIC}

La investigación en el área es un reflejo de la captación de fondos para la investigación por parte de los diferentes grupos. La falta de financiación industrial durante los últimos años, debida en buena parte a la crisis económica, unida a los temas prioritarios del anterior Programa Nacional de Ciencia y Tecnología de alimentos y el IV Programa $\mathrm{Marco}^{3}$ de la UE han definido las líneas de investigación. En la actualidad, la investigación en el área se lleva a cabo en cuatro grandes campos que se detallan a continuación, junto con sus correspondientes líneas de investigación e institutos implicados:

Modificaciones fisicas, químicas y bioquímicas de los alimentos en relación con la optimización de procesos. Se investiga en modificaciones e interacciones de los constituyentes de los alimentos durante los procesos tecnológicos (CEBAS, IATA, IFI, IFR, IGR, IIM, IPLA), fisiología y bioquímica de la maduración y conservación de alimentos (CEBAS, IATA, IFR, IGR), y bioquímica y microbiología de los procesos fermentativos (IATA. IFI, IFR, IGR, IIM, IPLA).

Tecnología de procesos. Se investiga en productos fermentados y eurados tradicionales (IATA, IFI, IFR. IGR, IPLA), nuevos procesos biotecnológicos (IATA, IPLA), conservación de productos en fresco (CEBAS, IATA, IFR, IGR, IPLA), control y optimización de procesos de obtención y transformación (IATA, IFI, IFR, IGR, IIM, IPLA), desarrollo de nuevos procesos y productos (IATA, IFR, IGR, IPLA) y tratamiento de aguas residuales y aprovechamiento de residuos (IATA, IGR).

Evaluación de calidad y seguridad de alimentos. Se investiga en el desarrollo de métodos analíticos avanzados (IATA, IFI, IIM), investigación prenormativa (IFI) y la caracterización de alimentos (CEBAS, IATA, IFI, IFR, IGR, IIM, IPLA).

Nutrición. Se investiga en la influencia de la composición y de los procesos sobre el valor nutritivo (IFI, IFR, IGR, INB) y la biodisponibilidad de nutrientes (INB).

En general los grupos de investigación del área no son grandes. Algunos institutos como el IATA o el IGR tienen un número elevado de grupos grandes con tres ó más investigadores. En el resto de institutos la situación difiere y es particularmente preocupante en algunos como el CEBAS o el INB. Como se indicó anteriormente, existen menos relaciones de las deseadas entre los distintos institutos. En lo relativo a temas lácteos las interacciones científicas son muy fuertes entre el IFI y el IFR y menos intensas de lo deseable con el IPLA. Hay coo- 
peraciones científicas en temas de postcosecha entre el IATA, el IFR y el IGR, y en temas de microbiología de alimentos entre el IATA y el IFI. Como se comentó anteriormente, sería deseable una mayor interacción en aras a la optimización de recursos. Además, las relaciones con otras áreas del conocimiento del CSIC son mínimas y sería importante incrementarlas.

Finalmente, señalamos las «fortalezas» y «debilidades» del área. Sin duda, los grupos del CSIC son fuertes en química, bioquímica, microbiología y biotecnología de alimentos. Sería necesario una mayor apuesta de investigación en nutrición y toxicología de alimentos, así como en ingeniería de procesos. Desgraciadamente no es fácil encontrar contratados postdoctorales formados en estos temas, dentro y fuera del CSIC. A pesar de ello, y en particular en nutrición, durante los últimos tres años se viene haciendo un esfuerzo continuado de captación de recursos humanos a través de la oferta de plazas de científico titular.

\section{Productividad del área de Ciencia y Tecnología de alimentos del CSIC}

La calidad de la investigación desarrollada en el área se puede considerar como muy aceptable. En este sentido, la lectura del factor de impacto SCI (science citation index) de las publicaciones del área puede ser constructiva, aunque al hacerlo hay que considerar la pluridisiciplinariedad del área y tener presente que aquellos grupos e institutos que investigan en temas relacionados con otras disciplinas científicas (bioquímica, biotecnología, nutrición) pueden publicar en revistas de mayor fuerza de impacto que aquellos que trabajan estrictamente en Tecnología de alimentos. Aun así, resulta revelador el constatar que desde el año 1994 se viene produciendo una subida de la media de factor de impacto SCI de las publicaciones del área (1.001 en el año 1994 a 1.333 en el año 1997). Esta subida es especialmente elevada en los casos del IATA y el IIM. El índice de impacto medio del área obtenido en el último año analizado es elevado, sobretodo si consideramos que la primera revista de artículos científicos del apartado «Food Science and Technology» del SCI tiene un factor de impacto 1.682. Todo ello indica un aumento continuado en la calidad científica de las publicaciones del área.

Frente a este aumento de la calidad también se detecta un aumento del número de publicaciones (193 en el año 1994 a 277 en el año 1997). Aunque a priori este hecho podría resultar atractivo, la falta 
de crecimiento en investigadores del área obliga a hacer una lectura mucho más detenida del resultado. Dicho análisis indica que, durante los últimos años y en muchos más grupos de lo deseable, se detecta un problema de aumento de número de las publicaciones en detrimento de la calidad. Los motivos que inducen esta tendencia pueden ser varios, pero sin duda uno de los más importantes es la extrema competencia en las oposiciones a científico titular y el mensaje lanzado por algunos investigadores del área sobre la importancia de la cantidad. Sin duda resulta necesario recordar desde estas líneas que la calidad siempre debe primar sobre la cantidad, y que cualquier valoración científica debe realizarse teniendo en cuenta de forma prioritaria la excelencia científica.

En el quinquenio 1994-97, los distintos grupos del área manejaron un total de 2438 millones de pesetas en recursos externos y 9072 millones de pesetas en recursos internos con una tasa de autofinanciación del $21,2 \%$. Esta última cifra es inferior a la media del CSIC del $29 \%$ para el mismo período de tiempo. Durante esos cinco años, los distintos grupos del área ingresaron un total de 2042 millones de pesetas en proyectos subvencionados por los distintos Programas Nacionales de la CICYT, el Programa Sectorial del Plan General del Conocimiento, las distintas Comunidades Autónomas y los Programas de agroalimentación de la UE. Si analizamos por Institutos, destaca el poder de captación de los investigadores del CEBAS y del IPLA (11.607 y 7.338 millones de pesetas/investigador respectivamente frente a una media del área de 6.609 millones de pesetas/investigador). Significativamente por debajo de la media se sitúa el INB con tan sólo 2.824 millones de pesetas por investigador.

El mayor foco de obtención de recursos del área ha sido el Programa Nacional de Tecnología de Alimentos. Si consideramos el quinquenio 1994-98, 109 de los 327 proyectos financiados por este Programa lo fueron a grupos del CSIC por un total de 1275 millones de pesetas (el $40 \%$ de los fondos financiados). La captación de fondos del Programa Nacional por los grupos del CSIC viene sufriendo una reducción progresiva durante los últimos años. Los motivos son claros. Por un lado no se ha producido un aumento del potencial científico del área mientras que los otros OPIs españoles, y en particular las distintas universidades a través de la creación de las licenciaturas de Ciencia y Tecnología de alimentos, han crecido en masa crítica investigadora. Por otro, no ha habido un aumento significativo de los fondos destinados a este Programa. Como consecuencia un mayor colectivo de científicos han concursado por los mismos fondos. Este hecho debe mover a la reflexión 


\section{Ciencia y Tecnología de alimentos}

y motivar a los distintos grupos del área a una preparación y presentación esmerada de sus proyectos al Plan Nacional, así como a la apuesta por otras fuentes de financiación, en particular los proyectos de la UE. En este sentido, la presencia de los grupos del CSIC en los programas de agroalimentación de la UE ha sido destacada. Baste citar que los distintos científicos del área han obtenido proyectos en el IV Programa Marco por valor de 1035 millones de pesetas. Sin duda, el CSIC ha sido el organismo español con mayor poder de captación de fondos en estas disciplinas.

La transferencia de resultados desde el área no ha sido, ni es, fácil. Resulta esperanzador el incremento de contratos con la industria detectado en los dos últimos años. En el período 1995-98 se han firmado un total de 159 contratos con industrias, casi todas ellas españolas, por un importe total ligeramente superior a los 584 millones de pesetas. La principal deficiencia detectada es la falta de un esquema de trabajo en las relaciones con la industria. Con frecuencia era el propio investigador quien negociaba dificultando el proceso. En la actualidad se pretende un mayor papel de la Oficina de Transferencia de Tecnología del CSIC que, en parte, se ha descentralizado creando grupos de trabajo en distintas Comunidades Autónomas como Andalucía o la Comunidad Valenciana. Dichos grupos están en permanente contacto con los distintos investigadores del área y representan a los investigadores en las negociaciones con los empresarios.

\section{El futuro de la investigación en Ciencia y Tecnología de alimentos en el CSIC.}

Un análisis simplista de la situación podría indicar que el futuro de la investigación en el área, debería venir marcado como hasta ahora, por las líneas prioritarias del nuevo Plan Nacional de Ciencia y Tecnología y el V Programa Marco de la UE. Dicha apuesta sería peligrosa si se realiza de forma excluyente. A este respecto, merece la pena recordar que la adecuación de las líneas actuales de investigación del área al IV Plan Nacional y al IV Programa Marco de la UE ha sido total, lo que ha situado a algunos grupos en una cierta situación de debilidad en algunas líneas de investigación, como por ejemplo la nutrición, la toxicología o la ingeniería de procesos, poco consideradas en los programas anteriores y fundamental en los actuales.

Dado el incremento de proyectos de los grupos del CSIC con la industria agroalimentaria española producido en los dos últimos años, 
también se hace prioritario el considerar las necesidades de los empresarios españoles. No es un ejercicio sencillo, ya que la evolución futura del sector agroalimentario español estará condicionada por una serie de factores complejos que podemos resumir en: i) la competencia derivada de la globalización de la economía, los acuerdos de la Organización Mundial del Comercio y la influencia de la moneda única en Europa; ii) la adecuación de la producción a las nuevas orientaciones de la política comunitaria en su Agenda 2000 que implica una menor influencia y apoyo de las políticas agraria y pesquera de la UE; iii) la alta concentración empresarial en la distribución comercial que requerirá una fuerte reestructuración de los sectores productivos; y iv) las nuevas demandas de los consumidores europeos sensibilizados con el binomio salud-alimentación y también con el medio ambiente. Muy probablemente, la consecuencia de todo ello será la búsqueda de competitividad, sobretodo en productos que requieran una acumulación de conocimientos y capacitaciones que los investigadores del CSIC pueden ofertar a los empresarios españoles.

A estos dos factores, los programas de investigación y las necesidades de la industria española, habría que añadir un tercer criterio que nos permita definir las líneas futuras de investigación. Se trata de las necesidades e intereses del consumidor español. Con frecuencia este hecho se olvida, cuando debería ser la premisa de investigación en el área. En cualquier disciplina tecnológica, pero más en Tecnología de alimentos, el consumidor tiene la última palabra al poder definir con sus apetencias las tendencias de mercado. Es evidente que el consumidor español, como el europeo, se encuentra sobretodo preocupado por la calidad higiénico-sanitaria y organoléptica de lo que come.

Tomando en consideración todas estas premisas, las líneas de investigación que se desarrollen en los próximos cinco años deberían englobar las actuales, sin que se vea necesario eliminar ninguna. Ahora bien, habría que reducir o reorientar alguna de ellas, y sobre todo potenciar otras que en estos momentos están bajo mínimos o simplemente no existen. Todo ello exigiría una serie de actuaciones que se detallan a continuación:

Líneas que mantener: «propiedades físicas, químicas y bioquímicas de los alimentos», «técnicas analíticas» y «Tecnología de procesos».

Líneas que incrementar: «biotecnología de alimentos», "Tecnología postcosecha», «análisis organoléptico de alimentos». En estos casos interesaría elevar ligeramente la masa crítica o abrir alguna de estas líneas en institutos donde no existen en la actualidad. 


\section{Ciencia y Tecnología de alimentos}

Líneas que potenciar: «Bioseguridad de alimentos (incluyendo Toxicología)», «Nutrición» e «Ingeniería de Procesos». En estas disciplinas las apuestas deberían ser firmes, intentando, dada la falta de contratados postdoctorales propios, captar contratados de otras áreas del conocimiento.

Sin olvidar la estabilización de los contratados postdoctorales de prestigio del área, esta apuesta exigiría la captación de investigadores de otras áreas del conocimiento. Asimismo sería aconsejable incrementar las interacciones científicas entre los distintos institutos y entre las distintas disciplinas del área. No es posible imaginar la producción de un alimento que no precise de un trabajo pluridisciplinar. Por ello resultaría necesario crear apuestas de investigación que pusieran en conexión los investigadores del área con los de otras áreas del conocimiento del CSIC. Ejemplos de estas conexiones podrían ser el establecimiento de acciones interáreas como «bioseguridad de alimentos» o «nutrición molecular» (área de Ciencia y Tecnología de alimentos y área de Biología y Biomedicina) o «ingeniería de alimentos» (área de Ciencia y Tecnología de alimentos y área de Física).

Para finalizar se hace necesario señalar el hecho de que tanto en el nuevo Plan Nacional de Ciencia y Tecnología de alimentos como en el V Programa Marco de la UE no exista un apartado específico de "Ciencia y Tecnología de alimentos", sino de "agroalimentación". Sin duda resulta difícil delimitar donde termina la agricultura o la ganadería y donde empieza la producción del alimento. Así, se ve necesario llevar a cabo la suma de los recursos humanos del CSIC en agroalimentación, poniendo en conexión sus efectivos humanos en agricultura y ganadería (área de Ciencias Agrarias), biología molecular de animales de granja y vegetales comestibles (área de Biología y Biomedicina) y Tecnología de alimentos (área de Ciencia y Tecnología de alimentos). Se trata de una necesidad estratégica cuyo horizonte está cada vez más cerca.

Notas

1 Las ideas expuestas en este artículo deben considerarse opinión personal del autor y nunca interpretarse como una postura oficial del CSIC.

2 Oficina de Ciencia y Tecnología del Gobierno Español. Plan Nacional de Ciencia y Tecnología (http://www.cicyt.es/ocyt/pnidi2000.htm).

3 V Programa Marco de la UE. (http://www.cicyt.es/sost/programa.htm). 